\title{
An acute immune response underlies the benefit of cardiac adult stem cell therapy
}

Ronald J. Vagnozzi ${ }^{1}$, Marjorie Maillet ${ }^{1}$, Michelle A. Sargent ${ }^{1}$, Hadi Khalii ${ }^{1}$, Anne Katrine Johansen ${ }^{1}$, Jennifer A. Schwanekamp ${ }^{2}$, Allen J. York ${ }^{1}$, Vincent Huang ${ }^{1}$, Matthias Nahrendorf ${ }^{3}$, Sakthivel Sadayappan ${ }^{2}$, Jeffery D. Molkentin ${ }^{1,4^{*}}$

${ }^{1}$ Department of Pediatrics, University of Cincinnati and Cincinnati Children's Hospital Medical Center, Cincinnati, OH, USA

${ }^{2}$ Department of Internal Medicine, Heart, Lung and Vascular Institute, University of Cincinnati, Cincinnati, OH, USA

${ }^{3}$ Center for Systems Biology, Department of Imaging, and Cardiovascular Research Center, Massachusetts General Hospital and Harvard Medical School, Boston, MA, USA.

${ }^{4}$ Howard Hughes Medical Institute, Cincinnati Children's Hospital Medical Center, Cincinnati, OH, USA

\section{*Correspondence:}

Jeffery D. Molkentin, PhD

Cincinnati Children's Hospital Medical Center,

Howard Hughes Medical Institute,

Molecular Cardiovascular Biology,

240 Albert Sabin Way, MLC 7020,

Cincinnati, OH 45229 USA.

jeff.molkentin@cchmc.org 
Clinical trials using adult stem cells to regenerate damaged heart tissue continue to this day ${ }^{1-3}$ despite ongoing questions of efficacy and a lack of mechanistic understanding of the underlying biologic effect ${ }^{4-6}$. The rationale for these cell therapy trials is derived from animal studies that show a modest but reproducible improvement in cardiac function in models of cardiac ischemic injury ${ }^{7-9}$. Here we examined the mechanistic basis for cell therapy in mice after ischemia/reperfusion (I/R) injury, and while heart function was enhanced, it was not associated with new cardiomyocyte production. Cell therapy improved heart function through an acute sterile immune response characterized by the temporal and regional induction of $\mathrm{CCR}^{+}$and $\mathrm{CX} 3 \mathrm{CR} 1^{+}$macrophages. Here we observed that intra-cardiac injection of 2 distinct types of progenitor cells, freeze/thaw-killed cells or a chemical inducer of the innate immune response similarly induced regional $C C R 2^{+}$and $C X 3 C R 1^{+}$macrophage accumulation and provided functional rejuvenation to the I/R-injured heart. Mechanistically, this selective macrophage response altered cardiac fibroblast activity and reduced border zone extracellular matrix (ECM) content and enhanced the mechanical properties of the injured area. The functional benefit of cardiac cell therapy is thus due to an acute inflammatory-based wound healing response that rejuvenates the mechanical properties of the infarcted area of the heart. Such results suggest a re-evaluation of strategies underlying cardiac cell therapy in current and planned human clinical trials.

Initial animal studies of adult stem or progenitor cell therapy for cardiac regeneration reported improved heart function with robust de novo myogenesis derived directly from the injected cells ${ }^{10-13}$. Many independent groups have since repeated these studies with an ever-increasing variety of adult stem cells and while functional improvement is reproducibly observed, nearly all have failed to observe new cardiomyocyte formation from these injected cells ${ }^{14-17}$. At the same time, clinical trials with adult stem cells in patients with acute myocardial infarction (MI) injury or decompensated heart failure have expanded worldwide over the past 17 years, with thousands of patients enrolled and over $\$ 1$ billion USD in funding utilized ${ }^{4,6}$. However, while results of these trials have been disappointing, this might simply reflect ineffective trial design because the true mechanistic basis of cell therapy remains unknown ${ }^{6}$. For example, more recent literature has shown that the vast majority of transplanted adult stem or progenitor cells simply die within days of delivery into the hearts of ischemia-injured animal models ${ }^{18,19}$, and as such it remains unclear how they might function in a therapeutic manner, although a paracrine hypothesis has been proposed whereby injected cells temporarily release protective growth factors or RNA species ${ }^{20-22}$.

Over 15 types of adult stem cells show some level of efficacy in cardiac regenerative studies of ischemic injury in animal models ${ }^{7,23}$. Here we focused on 2 primary types: fractionated bone marrow mononuclear cells (MNCs), which were the earliest and most heavily used cell type in clinical trials ${ }^{2}$, and cardiac mesenchymal cells from the heart that express the receptor tyrosine kinase c-Kit, which have been termed cardiac progenitor cells (CPCs) ${ }^{12,24}$. As we will describe, our major finding was that injection of these cell-types induces a sterile immune response in the heart, so we also examined the effect of injecting zymosan, a non-cellular and potent activator of the innate immune response ${ }^{25,26}$. MNCs and CPCs were isolated from mice expressing a constitutive membrane TdTomato (mTomato) fluorescent reporter and zymosan was conjugated to Alexa Fluor 594 to allow tracking in vivo by red fluorescence. Isolated MNCs were a heterogeneous cell population consisting of all major hematopoietic lineages although monocytes and granulocytes were the most predominant (Extended Data Fig. 1a). CPCs expressed mesenchymal cell surface markers but were negative for markers of hematopoietic or endothelial cells (Extended Data Fig. 1b).

We first asked whether cell injection could activate endogenous regenerative programs in the murine heart in the absence of acute damage or disease, reasoning that this approach would allow us to isolate the specific biologic effects of injecting cells into the heart apart from the profound cellular complexity of an infarction injury model. Eight-week-old male and female C57B//6J mice received 
intra-cardiac injection of either strain-matched MNCs, $10 \mu \mathrm{g}$ zymosan or saline. We chose a dose of 50,000 cells for these studies based on prior literature showing therapeutic benefit within this range ${ }^{7,8}$, while our zymosan dosing in adult mice was extrapolated from a recent report using zymosan injection into neonatal mouse hearts ${ }^{27}$. Injected material was delivered across 3 defined regions along the anterior wall of the left ventricle, mirroring the region of the heart most affected by MI (Fig. 1a). Importantly, none of the injected mTomato-labeled MNCs transdifferentiated into cardiomyocytes or endothelial cells (not shown). Distinct histological foci of acute local hypercellularity were observed with both cell and zymosan injection, as examined by confocal microscopy from heart sections 3 days or 2 weeks post-injection, suggestive of an inflammatory response (Fig. 1b). Indeed, activated CD68 ${ }^{+}$macrophages were significantly increased specifically within the area of injection at 3 days, with a diminishing effect by 2 weeks as the cells or zymosan were cleared (Fig. $1 \mathrm{~b}, \mathrm{c})$. We also examined neutrophil levels by flow cytometry from dissociated hearts at 3 days, but we did not observe a significant increase with cell or zymosan injection (Extended Data Fig. 1c).

To further profile the induction of macrophages with MNCs or zymosan injection, we employed a genetic approach in mice to differentially label $\mathrm{CCR} 2^{+}$and $\mathrm{CX} 3 \mathrm{CR} 1^{+}$macrophages with either red $\left(\right.$ Ccr2-RFP $\left.{ }^{28}\right)$ or green $\left(C \times 3 c r 1-G F P^{29}\right)$ fluorescence. CCR2 and CX3CR1 regulate the recruitment and/or activation of circulating monocyte-derived or tissue-resident macrophages, respectively ${ }^{30-33}$, and have been established as markers to broadly distinguish pro-inflammatory monocyte-derived macrophages $\left(\mathrm{CCR} 2^{+}\right)$versus pro-healing macrophages $\left(\mathrm{CX} 3 \mathrm{CR} 1^{+} \mathrm{CCR} 2^{-}\right)^{34-39}$. We delivered unlabeled MNCs or zymosan into 8-week-old Ccr2-RFP x Cx3cr1-GFP mice by intracardiac injection (Fig. 1d). Uninjured adult (non-injected) hearts showed GFP ${ }^{+}\left(\mathrm{CX} 3 \mathrm{CR} 1^{+}\right)$tissue-resident macrophages throughout the myocardium while $\mathrm{RFP}^{+}\left(\mathrm{CCR} 2^{+}\right)$macrophages were largely absent (Fig. 1e). After 1 day, areas of MNC and zymosan injection in the heart showed a robust and highly localized influx of $\mathrm{CCR} 2^{+}$macrophages within the injection site while CX3CR1 ${ }^{+}$macrophages were largely restricted to the periphery of the injected area (Fig 1e). By 3 days these CX3CR $1^{+}$ macrophages expanded within the injection area along with CCR $2^{+}$macrophages, but by 7 days levels of both macrophage types were reduced, indicative of a biphasic and temporal response. Flow cytometry analysis from these mice at 3 days also indicated a shift in overall macrophage subtype content from a largely CX3CR $1^{+}$population in the naïve state to a mix of CCR2 ${ }^{+}$and $\mathrm{CCR} 2^{+}$

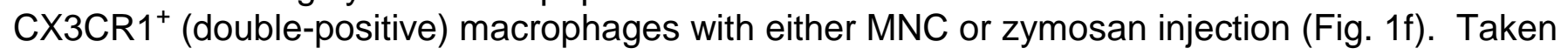
together these data suggest that the principal endogenous cellular response to intra-cardiac cell therapy or zymosan injection is an acute and localized inflammation through biphasic involvement of $\mathrm{CCR}^{+}$and $\mathrm{CX} 3 \mathrm{CR} 1^{+}$macrophages that is largely cleared within 1 week.

One aspect of the proposed paracrine hypothesis of cell therapy introduced above is that the injected cells secrete effectors that cause endogenous cardiomyocytes to proliferate ${ }^{20,21,40}$, which we examined by immunohistochemistry from hearts injected with MNCs or zymosan (Fig. 2a). We used an antibody against PCM-1 to specifically mark cardiomyocyte nuclei ${ }^{41}$ and Ki67 to label nuclei with cell cycle activity (Fig. 2b). No appreciable increase in cardiomyocyte cell cycle activity was observed versus saline-injected controls, either at areas of injection or distally across the entire tissue (Fig. 2c). Another proposed effect of cell therapy is the activation of endogenous stem or progenitor cells, in particular cardiac-resident stem cells expressing $\mathrm{c}-\mathrm{Kit}^{21,40}$. We and others ${ }^{42-45}$ have employed genetic lineage tracing of $\mathrm{c}-\mathrm{Kit}^{+}$cells in vivo and found that their endogenous contribution to cardiomyogenesis is negligible even after injury. Here we used tamoxifen-inducible Kit ${ }^{\mathrm{MerCreMer} /+} \times \mathrm{R}^{-}$ eGFP lineage tracing mice to directly examine new cardiomyocyte generation from endogenous $\mathrm{c}-\mathrm{Kit}^{+}$ cells. Tamoxifen was administered over 6 weeks allowing for greater cumulative eGFP labeling. For these experiments we also injected cardiac progenitor cells (CPCs) that were isolated from the heart and selected for c-Kit positivity and then expanded in culture (CPCs), in addition to MNCs or zymosan (Fig. 2d). We observed very rare single c-Kit ${ }^{+}$-derived (eGFP ${ }^{+}$) cardiomyocytes in all treatment groups at a physiologically insignificant amount that was not greater with injected MNCs, CPCs, or zymosan versus saline (Fig. 2e, f). These data demonstrate that stem or progenitor cell injection does 
not cause endogenous cardiomyocyte proliferation or the induction of cardiomyocytes from endogenous c-Kit ${ }^{+}$progenitor cells.

We also assessed the formation of new endogenous endothelial cells from the $\mathrm{C}_{-} \mathrm{Kit}^{+}$lineage in the hearts of $\mathrm{Kit}^{\mathrm{MerCreMer/+}} \times$ R-eGFP mice (Fig. 2g-j). Two weeks after injection, eGFP ${ }^{+}$endothelial cells were significantly increased at the injection sites of zymosan-treated (Fig. 2h, 2i) hearts. In contrast we observed a non-significant increase with MNC or CPC injection (Fig. 2g, 2i) and by 6 weeks only zymosan injection still gave a significant increase in endothelial cells (Fig. 2j). However, zymosan persists the longest within the heart while CPCs and MNCs are essentially cleared by 2 weeks, (Fig. 2g, 2h). None of the treatments increased c-Kit ${ }^{+}$-derived endothelial cells in the distal areas of the heart, suggesting localized induction in areas of active inflammation. Thus, the acute inflammatory response associated with cell therapy or zymosan injection could have a mild cardioprotective effect through a transient increase in new capillary formation (see below).

We next investigated whether cell therapy or acute inflammation with zymosan could positively impact the function of the mouse heart following MI injury due to prolonged ischemia/reperfusion (I/R). We injected either 150,000 total strain-matched MNCs, CPCs, $20 \mu \mathrm{g}$ zymosan or saline (doses increased to account for exacerbated cell loss in the injured heart) on each side of the heart's infarct border zone in C57BI/6J mice, 1 week post-I/R injury (Fig. 3a). Importantly, cell or zymosan injection into uninjured hearts did not alter LV structure or function (Extended Data Fig. 2 a-f). Injection of MNCs, CPCs or zymosan each significantly improved post-l/R cardiac function at $2 \mathrm{w}$ post-injection (3w post-I/R) as measured by fractional shortening (Fig. 3b), and this was associated with improvements in left ventricular end-systolic volume (LVESV; Extended Data Fig. 3a). In contrast, cell or zymosan therapy showed no change in end-diastolic volume (LVEDV; Extended Data Fig. 3b) or heart rate (Extended Data Fig. 3c) across any of the treatment groups at 2w post-therapy, suggesting no effect on ventricular remodeling, but instead that contractile properties of the heart were directly impacted. Importantly, the functional benefit persisted for at least 8w after injection, as indicated by increased fractional shortening in MNC or zymosan-treated mice (Fig. 3c). By 8w after injection, saline-treated I/R hearts also began to show ventricular dilation as indicated by a significant increase in LVEDV, but this was attenuated in the hearts of MNC-treated mice (data not shown).

Our data indicated that the predominant effect of intracardiac cell therapy was localized and biphasic action of $C C R 2^{+}$followed by $C X 3 C R 1^{+}$macrophages, which was recapitulated with zymosan. To more specifically examine whether this inflammatory response was a mediator of postI/R rejuvenation with cell therapy, we first treated mice with cyclosporine $A$ (CsA), a broad-spectrum immunosuppressant, starting 1 day prior to cell injection. MNCs were used as they are the overwhelming cell-type used in human clinical trials ${ }^{1,2}$. Remarkably, CsA abrogated the restorative effects on cardiac function seen with MNC or zymosan injection after I/R injury (Fig. 3d), indicating that the immune response was required for the observed benefit. In addition, we injected freeze-thaw killed MNCs to address the paracrine hypothesis, and remarkably, dead cell debris similarly improved cardiac function post-I/R, further suggesting that the acute sterile immune response is a primary mechanism of action for cell therapy (Extended Data Fig. 3d).

Cardiac I/R injury itself is associated with a robust and temporally regulated recruitment of discrete myeloid cell populations ${ }^{34,46,47}$, which we also observed using our Ccr2-RFP x Cx3cr1-GFP mice. (Fig. $3 e$ ). Of note, this sequential expansion of $\mathrm{CCR}^{+}$followed by $\mathrm{CX} 3 \mathrm{CR} 1^{+}$macrophages in the developing scar and infarct border zone was reminiscent of the localized pattern we observed with cell therapy or zymosan injection into naïve hearts, suggesting that cell therapy injection simply invokes another round of acute wound healing. To test this, we employed $\mathrm{Ccr}^{-/-}$or $\mathrm{C} \times 3 \mathrm{cr} 1^{-/-}$genetargeted mice. Although initial infarct sizes post-l/R were not different among $C \mathrm{cr} 2^{-/-}$or $\mathrm{C} \times 3 \mathrm{cr} 1^{-/-}$mice or strain-matched wild-type controls (not shown), Ccr2 deficiency significantly improved cardiac function after I/R (Fig. 3h), consistent with previous reports ${ }^{31,48,49}$. Moreover, cell therapy by MNC injection in mice lacking Ccr2 imparted no further functional benefit, given the already enhanced state of cardiac healing after I/R (Fig.3h). Loss of Ccr2 showed a reduction in overall CD68 ${ }^{+}$cell content in 
the post-I/R heart with or without cell therapy, consistent with prior studies demonstrating that targeting circulating $\mathrm{CCR}^{+}$monocytes/macrophages can reduce inflammation in the heart ${ }^{31,49}$. By comparison, Cx3cr1 null mice lacking tissue resident macrophage activity showed left ventricular dysfunction after I/R injury that was similar to wild-type controls, but these mice no longer benefitted from MNC therapy and showed a much greater total inflammatory response (Fig. 3h, i). This result suggests that endogenous tissue resident macrophages are necessary for a protective healing response associated with cell therapy. The increase in total inflammation in the I/R injured hearts of Cx3cr1 null mice is consistent with recent data whereby these tissue-resident macrophages play an immunomodulatory role, dampening excess inflammation and promoting healing ${ }^{34,50}$.

Since cell therapy or zymosan injection produced only a transient increase in endothelial cell content in the heart it seemed unlikely to be the primary mechanism for protection. Thus, we hypothesized that a more fundamental mechanism was at play, such as an alteration in the properties of the ECM within the injury area and border zone. While overall infarct size in MNC-treated mice was unchanged versus controls (not shown), fibrotic burden specifically in the peri-infarct border zone was significantly decreased with MNC cell therapy (Fig. 4a, b). This was also observed with injection of non-viable MNCs, suggesting that it was primarily due to immunoreactivity and not active paracrine signaling (Fig. 4c). Tissue strips from the infarct region of saline or MNC-injected hearts were isolated and subjected to passive force-tension stress analysis. Remarkably, infarct strips from MNCinjected hearts produced a significantly greater change in passive force over increasing stretch (change in initial length [ $\left.\mathrm{L}_{0}\right]$; Fig. 4d). This was associated with a decrease in gene expression levels of several ECM and matricellular components in MNC versus saline treated hearts post-I/R (Fig. 4e). Together these data indicate that infarcts from MNC-injected hearts have improved mechanical properties and a reduction in fibrotic content versus saline controls. We also repeated the forcelengthening assay on infarct strips from post-I/R hearts injected with zymosan, which showed that these infarcts had an even larger improvement in passive force dynamics compared with either saline or MNC treatment (Extended Data Fig. 4a).

To further define the mechanism, we performed a series of in vitro experiments using purified macrophages to determine how the acute induction of inflammation with cell therapy might impart structural and functional changes to the post-I/R region of the heart. We first isolated either bone marrow-derived (BMDM) or peritoneal ( $P M)$ macrophages from naïve mice, which have been used as broad models for circulating monocyte-derived or tissue-resident macrophages, respectively ${ }^{51,52}$. We cultured these macrophages on pre-fabricated collagen patches and then imaged the patches with second harmonic generation microscopy to examine collagen organization. Addition of macrophages to these patches, which lack fibroblasts or any other cell type, resulted in dramatic remodeling of the collagen matrix (Fig. 4f). This effect was highly distinct between BMDM, which produced wide folds throughout the patch, versus PM, which reorganized the matrix into thinner and interlaced sheets. To test whether a similar reorganization of collagen via macrophage activity was occurring in our cell or zymosan-treated hearts in vivo, we performed histological analysis on post-I/R hearts from Ccr2-RFP $x$ Cx3cr1-GFP mice that received MNCs or zymosan, using a recently described collagen hybridizing peptide $\left(\mathrm{CHP}^{53}\right)$ that specifically detects immature or denatured collagen (Extended Data Fig. 4b). Hearts from MNC or zymosan treated mice showed a CHP reactivity that was coincident with enhanced presence of $\mathrm{CCR} 2^{+}$and $\mathrm{CX} 3 \mathrm{CR} 1^{+}$macrophages (Extended Data Fig. 4c), suggesting that sites of active inflammation within the areas of cell or zymosan injection were undergoing greater ECM remodeling. To address whether these activated macrophages could also be directly impacting cardiac fibroblasts, we purified $\mathrm{CCR} 2^{+}$or $\mathrm{CX} 3 \mathrm{CR} 1^{+}$macrophages from hearts at 7 days post-I/R (Fig. $4 \mathrm{~g}$ ) and cultured them for $72 \mathrm{~h}$ with freshly isolated cardiac fibroblasts. Gene expression analysis of these fibroblasts by RT-PCR revealed that CCR2 ${ }^{+}$macrophages imparted an activating signal, as indicated by increased fibroblast expression of smooth muscle a-actin (Acta2 / aSMA), lysyl oxidase (Lox), and collagen 1 alpha 2 (Col1a2) (Fig. 4h, i, k). In contrast, CX3CR1 ${ }^{+}$macrophages slightly reduced expression of these genes but increased fibroblast expression of connective tissue growth 
factor (Ctgf) (Fig. 4j), which has been associated with maintenance of the ECM as well as angiogenesis ${ }^{54}$. Taken together, these results demonstrate that acute localized inflammatory stimuli from $\mathrm{CCR} 2^{+}$and tissue-resident $\mathrm{CX} 3 \mathrm{CR} 1^{+}$macrophages due to cell therapy improves the passive mechanical properties of the injured area by influencing the activity of cardiac fibroblasts.

Two of the most highly used adult stem cells, MNCs and CPCs, improved cardiac function in mice when delivered directly into both lateral sides of the infarct border zone, in agreement with previous data ${ }^{10,13}$. However, the same improvement was obtained with zymosan, a member of the pathogen-associated molecular pattern (PAMP) family that primarily acts via toll-like receptors to induce acute inflammation ${ }^{26}$. The observed functional benefit required macrophage-mediated inflammation, as shown using genetic or pharmacological inhibition. Most progenitor cells injected into the heart rapidly die $7,8,18,19$, and dying cells release damage-associated molecular patterns (DAMPs), cellular fragments that like PAMPs, are highly immunogenic ${ }^{26,55,56}$. Indeed, injection of freeze-thawed killed MNCs, containing DAMPs but incapable of active paracrine factor production, imparted equivalent benefit as live cells. However, our results do not rule out an additive effect of paracrine factors or miRNAs released over time from living injected cells.

The transitory and localized nature of this inflammatory response is likely crucial in producing a benefit, as excessive and chronic inflammation throughout the heart appears to be universally pathological ${ }^{56,57}$. In this context the very low retention and rapid clearance of injected adult stem cells may actually be advantageous. These observations may also explain why direct injection into the parenchyma of the heart, as done in the majority of animal studies ${ }^{7-9}$, is universally efficacious while pre-clinical studies using systemic vascular infusion of progenitor cells as performed in the majority of human clinical trials have shown mixed results ${ }^{4,6,58,59}$. Indeed, it is uncertain how systemic vascular delivery of progenitor cells could provide benefit to the heart given the mechanism of action we proposed here, as the vast majority of infused cells do not take up residence in the heart nor persist in the circulatory system ${ }^{60,61}$.

Our study was designed to examine the mechanistic basis of adult cardiac stem cell therapy given the new consensus that direct cardiomyocyte regeneration is no longer a tenable mechanism $^{17,62}$ and that the adult mammalian heart is largely non-regenerative and does not contain a resident stem cell population ${ }^{63-65}$. As suggested 10 years ago ${ }^{66}$, we observed that the acute inflammatory response is the overwhelming mechanism of benefit behind cell therapy to the post-MI injured heart. Previous studies using severe genetically immunodeficient animals have also demonstrated that the benefit of injecting a type of adult bone marrow cell in the heart post-MI was lost $^{67}$. We identified a unique mechanism whereby stimulating the intrinsic wound healing cascade and sequential activity of circulating $\mathrm{CCR} 2^{+}$followed by $\mathrm{CX} 3 \mathrm{CR} 1^{+}$tissue resident macrophages positively impacted the ECM around and within the infarcted region of the heart, such that functional performance was significantly improved. This acute inflammatory response provides instruction to resident fibroblasts in modifying the border zone ECM and its mechanical properties ${ }^{46,68-70}$. The dynamics and cell-specific regulation of inflammation by macrophage subsets in the heart is a growing area of study ${ }^{35,36,50,70}$, such that the timing and rapid clearance of immune cells is also key to proper healing ${ }^{71}$. Hence, it might be warranted to re-evaluate current and planned cell therapy-based clinical trials to maximize the effects of the most prevalent underlying biologic mechanism of action demonstrated here.

\section{METHODS SUMMARY (also see supplementary methods)}

Candidate cellular therapeutics (MNCs or CPCs) were isolated from mice with a genetically-encoded fluorescent mTomato reporter and delivered by intra-cardiac injection, either at baseline or after I/R injury, into strain-matched animals that were either wild-type, genetically null for macrophage subtypes, or carrying Kit-MerCreMer and Rosa26-eGFP transgenes (R26-eGFP, for genetic lineage tracing). Cardiac function, inflammation, fibrosis, and cellular regeneration were assessed by echocardiography, immunohistochemistry, or flow cytometry. See Supplementary Methods for more 
detailed information and Supplementary Tables 1 and 2 for description of primary antibodies and RTPCR primers used.

\section{Supplementary Information:}

Extended Data Figure 1

Extended Data Figure 2

Extended Data Figure 3

Extended Data Figure 4

Supplementary Table 1

Supplementary Table 2

Supplementary Methods

\section{Acknowledgments:}

This work was supported by grants from the National Institutes of Health to J.D.M., S.S., and M.N. J.D.M. was also supported by the Howard Hughes Medical Institute. R.J.V. was supported by a National Research Service Award from the NIH (F32 HL128083). All flow cytometric data were acquired using equipment maintained by the Research Flow Cytometry Core in the Division of Rheumatology at Cincinnati Children's Hospital Medical Center.

\section{Author Contributions:}

J.D.M. and R.J.V. conceived the study. R.J.V., M.M., M.A.S., H.K., A.K.J., J.A.S., A.J.Y. and V.H. performed experiments and generated all the data shown in the manuscript. S.S. provided oversight and technical help along with J.A.S. in measuring myocardial scar mechanical properties. M.N. provided theoretical assessment of the project and advice in experimental design.

\section{Conflict of Interest or Competing Financial Interest:}

None

\section{References}

1 Madonna, R. et al. Position Paper of the European Society of Cardiology Working Group Cellular Biology of the Heart: cell-based therapies for myocardial repair and regeneration in ischemic heart disease and heart failure. Eur Heart J 37, 1789-1798, doi:10.1093/eurheartj/ehw113 (2016).

2 Nguyen, P. K., Rhee, J. W. \& Wu, J. C. Adult Stem Cell Therapy and Heart Failure, 2000 to 2016: A Systematic Review. JAMA Cardiol 1, 831-841, doi:10.1001/jamacardio.2016.2225 (2016).

3 Fernandez-Aviles, F. et al. Global position paper on cardiovascular regenerative medicine. Eur Heart $J$ 38, 2532-2546, doi:10.1093/eurheartj/ehx248 (2017).

$4 \quad$ A futile cycle in cell therapy. Nat Biotechnol 35, 291, doi:10.1038/nbt.3857 (2017).

5 Marks, P. W., Witten, C. M. \& Califf, R. M. Clarifying Stem-Cell Therapy's Benefits and Risks. $N$ Engl J Med 376, 1007-1009, doi:10.1056/NEJMp1613723 (2017).

6 Epstein, J. A. A Time to Press Reset and Regenerate Cardiac Stem Cell Biology. JAMA Cardiol, doi:10.1001/jamacardio.2018.4435 (2018).

7 Zwetsloot, P. P. et al. Cardiac Stem Cell Treatment in Myocardial Infarction: A Systematic Review and Meta-Analysis of Preclinical Studies. Circ Res 118, 1223-1232, doi:10.1161/CIRCRESAHA.115.307676 (2016).

8 Golpanian, S. et al. Concise Review: Review and Perspective of Cell Dosage and Routes of Administration From Preclinical and Clinical Studies of Stem Cell Therapy for Heart Disease. Stem Cells Transl Med 5, 186-191, doi:10.5966/sctm.2015-0101 (2016).

9 Tompkins, B. A. et al. Preclinical Studies of Stem Cell Therapy for Heart Disease. Circ Res 122, 10061020, doi:10.1161/CIRCRESAHA.117.312486 (2018). 
Orlic, D. et al. Transplanted adult bone marrow cells repair myocardial infarcts in mice. Ann N Y Acad Sci 938, 221-229; discussion 229-230 (2001).

11 Orlic, D. et al. Bone marrow cells regenerate infarcted myocardium. Nature 410, 701-705, doi:10.1038/35070587 (2001).

12 Beltrami, A. P. et al. Adult cardiac stem cells are multipotent and support myocardial regeneration. Cell 114, 763-776 (2003).

13 Amado, L. C. et al. Cardiac repair with intramyocardial injection of allogeneic mesenchymal stem cells after myocardial infarction. Proc Natl Acad Sci U S A 102, 11474-11479, doi:10.1073/pnas.0504388102 (2005).

14 Murry, C. E. et al. Haematopoietic stem cells do not transdifferentiate into cardiac myocytes in myocardial infarcts. Nature 428, 664-668, doi:10.1038/nature02446 (2004).

15 Balsam, L. B. et al. Haematopoietic stem cells adopt mature haematopoietic fates in ischaemic myocardium. Nature 428, 668-673, doi:10.1038/nature02460 (2004).

16 Tang, X. L. et al. Long-Term Outcome of Administration of c-kit(POS) Cardiac Progenitor Cells After Acute Myocardial Infarction: Transplanted Cells Do not Become Cardiomyocytes, but Structural and Functional Improvement and Proliferation of Endogenous Cells Persist for at Least One Year. Circ Res 118, 1091-1105, doi:10.1161/CIRCRESAHA.115.307647 (2016).

17 Eschenhagen, T. et al. Cardiomyocyte Regeneration: A Consensus Statement. Circulation 136, 680-686, doi:10.1161/CIRCULATIONAHA.117.029343 (2017).

18 Hong, K. U. et al. A highly sensitive and accurate method to quantify absolute numbers of c-kit+ cardiac stem cells following transplantation in mice. Basic Res Cardiol 108, 346, doi:10.1007/s00395-0130346-0 (2013).

19 Pons, J. et al. Combining angiogenic gene and stem cell therapies for myocardial infarction. J Gene Med 11, 743-753, doi:10.1002/jgm.1362 (2009).

20 Sanganalmath, S. K. \& Bolli, R. Cell therapy for heart failure: a comprehensive overview of experimental and clinical studies, current challenges, and future directions. Circ Res 113, 810-834, doi:10.1161/CIRCRESAHA.113.300219 (2013).

21 Mirotsou, M., Jayawardena, T. M., Schmeckpeper, J., Gnecchi, M. \& Dzau, V. J. Paracrine mechanisms of stem cell reparative and regenerative actions in the heart. J Mol Cell Cardiol 50, 280-289, doi:10.1016/j.yjmcc.2010.08.005 (2011).

22 Glembotski, C. C. Expanding the Paracrine Hypothesis of Stem Cell-Mediated Repair in the Heart: When the Unconventional Becomes Conventional. Circ Res 120, 772-774, doi:10.1161/CIRCRESAHA.116.310298 (2017).

23 Cambria, E. et al. Translational cardiac stem cell therapy: advancing from first-generation to nextgeneration cell types. NPJ Regen Med 2, 17, doi:10.1038/s41536-017-0024-1 (2017).

24 Ellison, G. M. et al. Adult c-kit(pos) cardiac stem cells are necessary and sufficient for functional cardiac regeneration and repair. Cell 154, 827-842, doi:10.1016/j.cell.2013.07.039 (2013).

25 Pillemer, L., Blum, L., Pensky, J. \& Lepow, I. H. The requirement for magnesium ions in the inactivation of the third component of human complement (C'3) by insoluble residues of yeast cells (zymosan). J Immunol 71, 331-338 (1953).

26 Piccinini, A. M. \& Midwood, K. S. DAMPening inflammation by modulating TLR signalling. Mediators Inflamm 2010, doi:10.1155/2010/672395 (2010).

27 Han, C. et al. Acute inflammation stimulates a regenerative response in the neonatal mouse heart. Cell Res 25, 1137-1151, doi:10.1038/cr.2015.110 (2015).

28 Saederup, N. et al. Selective chemokine receptor usage by central nervous system myeloid cells in CCR2-red fluorescent protein knock-in mice. PLoS One 5, e13693, doi:10.1371/journal.pone.0013693 (2010).

29 Jung, S. et al. Analysis of fractalkine receptor CX(3)CR1 function by targeted deletion and green fluorescent protein reporter gene insertion. Mol Cell Biol 20, 4106-4114 (2000). 
30 Dutta, P. et al. Myocardial Infarction Activates CCR2(+) Hematopoietic Stem and Progenitor Cells. Cell Stem Cell 16, 477-487, doi:10.1016/j.stem.2015.04.008 (2015).

31 Leuschner, F. et al. Therapeutic siRNA silencing in inflammatory monocytes in mice. Nat Biotechnol 29, 1005-1010, doi:10.1038/nbt.1989 (2011).

32 Bajpai, G. et al. The human heart contains distinct macrophage subsets with divergent origins and functions. Nat Med 24, 1234-1245, doi:10.1038/s41591-018-0059-x (2018).

33 Pinto, A. R. et al. An abundant tissue macrophage population in the adult murine heart with a distinct alternatively-activated macrophage profile. PLoS One 7, e36814, doi:10.1371/journal.pone.0036814 (2012).

34 Nahrendorf, M. et al. The healing myocardium sequentially mobilizes two monocyte subsets with divergent and complementary functions. J Exp Med 204, 3037-3047, doi:10.1084/jem.20070885 (2007).

35 Epelman, S. et al. Embryonic and adult-derived resident cardiac macrophages are maintained through distinct mechanisms at steady state and during inflammation. Immunity 40, 91-104, doi:10.1016/j.immuni.2013.11.019 (2014).

36 Lavine, K. J. et al. Distinct macrophage lineages contribute to disparate patterns of cardiac recovery and remodeling in the neonatal and adult heart. Proc Natl Acad Sci U S A 111, 16029-16034, doi:10.1073/pnas.1406508111 (2014).

37 Yona, S. et al. Fate mapping reveals origins and dynamics of monocytes and tissue macrophages under homeostasis. Immunity 38, 79-91, doi:10.1016/j.immuni.2012.12.001 (2013).

38 Thomas, G., Tacke, R., Hedrick, C. C. \& Hanna, R. N. Nonclassical patrolling monocyte function in the vasculature. Arterioscler Thromb Vasc Biol 35, 1306-1316, doi:10.1161/ATVBAHA.114.304650 (2015).

39 Stremmel, C. et al. Yolk sac macrophage progenitors traffic to the embryo during defined stages of development. Nat Commun 9, 75, doi:10.1038/s41467-017-02492-2 (2018).

40 Gnecchi, M., Zhang, Z., Ni, A. \& Dzau, V. J. Paracrine mechanisms in adult stem cell signaling and therapy. Circ Res 103, 1204-1219, doi:10.1161/CIRCRESAHA.108.176826 (2008).

41 Bergmann, O. et al. Identification of cardiomyocyte nuclei and assessment of ploidy for the analysis of cell turnover. Exp Cell Res 317, 188-194, doi:10.1016/j.yexcr.2010.08.017 (2011).

42 van Berlo, J. H. et al. c-kit+ cells minimally contribute cardiomyocytes to the heart. Nature 509, 337341, doi:10.1038/nature13309 (2014).

43 Sultana, N. et al. Resident c-kit(+) cells in the heart are not cardiac stem cells. Nat Commun 6, 8701, doi:10.1038/ncomms9701 (2015).

44 Hatzistergos, K. E. et al. cKit+ cardiac progenitors of neural crest origin. Proc Natl Acad Sci U S A 112, 13051-13056, doi:10.1073/pnas.1517201112 (2015).

45 Liu, Q. et al. Genetic lineage tracing identifies in situ Kit-expressing cardiomyocytes. Cell Res 26, 119130, doi:10.1038/cr.2015.143 (2016).

46 Prabhu, S. D. \& Frangogiannis, N. G. The Biological Basis for Cardiac Repair After Myocardial Infarction: From Inflammation to Fibrosis. Circ Res 119, 91-112, doi:10.1161/CIRCRESAHA.116.303577 (2016).

47 Epelman, S., Liu, P. P. \& Mann, D. L. Role of innate and adaptive immune mechanisms in cardiac injury and repair. Nat Rev Immunol 15, 117-129, doi:10.1038/nri3800 (2015).

48 Kaikita, K. et al. Targeted deletion of CC chemokine receptor 2 attenuates left ventricular remodeling after experimental myocardial infarction. Am J Pathol 165, 439-447, doi:10.1016/S00029440(10)63309-3 (2004).

49 Grisanti, L. A. et al. beta2-Adrenergic receptor-dependent chemokine receptor 2 expression regulates leukocyte recruitment to the heart following acute injury. Proc Natl Acad Sci U S A 113, 15126-15131, doi:10.1073/pnas.1611023114 (2016).

50 Pinto, A. R., Godwin, J. W. \& Rosenthal, N. A. Macrophages in cardiac homeostasis, injury responses and progenitor cell mobilisation. Stem Cell Res 13, 705-714, doi:10.1016/j.scr.2014.06.004 (2014). 
51 Zhang, X., Goncalves, R. \& Mosser, D. M. The isolation and characterization of murine macrophages. Curr Protoc Immunol Chapter 14, Unit 14 11, doi:10.1002/0471142735.im1401s83 (2008).

52 Davies, L. C., Jenkins, S. J., Allen, J. E. \& Taylor, P. R. Tissue-resident macrophages. Nat Immunol 14, 986-995, doi:10.1038/ni.2705 (2013).

53 Hwang, J. et al. In Situ Imaging of Tissue Remodeling with Collagen Hybridizing Peptides. ACS Nano 11, 9825-9835, doi:10.1021/acsnano.7b03150 (2017).

54 Brigstock, D. R. Regulation of angiogenesis and endothelial cell function by connective tissue growth factor (CTGF) and cysteine-rich 61 (CYR61). Angiogenesis 5, 153-165 (2002).

55 Zhang, W. et al. Necrotic myocardial cells release damage-associated molecular patterns that provoke fibroblast activation in vitro and trigger myocardial inflammation and fibrosis in vivo. J Am Heart Assoc 4, e001993, doi:10.1161/JAHA.115.001993 (2015).

56 Mann, D. L. Innate immunity and the failing heart: the cytokine hypothesis revisited. Circ Res 116, 1254-1268, doi:10.1161/CIRCRESAHA.116.302317 (2015).

57 Nahrendorf, M. \& Swirski, F. K. Innate immune cells in ischaemic heart disease: does myocardial infarction beget myocardial infarction? Eur Heart J 37, 868-872, doi:10.1093/eurheartj/ehv453 (2016). Perin, E. C. et al. Comparison of intracoronary and transendocardial delivery of allogeneic mesenchymal cells in a canine model of acute myocardial infarction. J Mol Cell Cardiol 44, 486-495, doi:10.1016/j.yjmcc.2007.09.012 (2008).

59 Kanelidis, A. J., Premer, C., Lopez, J., Balkan, W. \& Hare, J. M. Route of Delivery Modulates the Efficacy of Mesenchymal Stem Cell Therapy for Myocardial Infarction: A Meta-Analysis of Preclinical Studies and Clinical Trials. Circ Res 120, 1139-1150, doi:10.1161/CIRCRESAHA.116.309819 (2017).

60 Hofmann, M. et al. Monitoring of bone marrow cell homing into the infarcted human myocardium. Circulation 111, 2198-2202, doi:10.1161/01.CIR.0000163546.27639.AA (2005).

61 Hou, D. et al. Radiolabeled cell distribution after intramyocardial, intracoronary, and interstitial retrograde coronary venous delivery: implications for current clinical trials. Circulation 112, I150-156, doi:10.1161/CIRCULATIONAHA.104.526749 (2005).

62 Vagnozzi, R. J., Molkentin, J. D. \& Houser, S. R. New Myocyte Formation in the Adult Heart: Endogenous Sources and Therapeutic Implications. Circ Res 123, 159-176, doi:10.1161/CIRCRESAHA.118.311208 (2018).

63 Cai, C. L. \& Molkentin, J. D. The Elusive Progenitor Cell in Cardiac Regeneration: Slip Slidin' Away. Circ Res 120, 400-406, doi:10.1161/CIRCRESAHA.116.309710 (2017).

$64 \mathrm{Li}$, Y. et al. Genetic Lineage Tracing of Non-Myocyte Population by Dual Recombinases. Circulation, doi:10.1161/CIRCULATIONAHA.118.034250 (2018).

65 Kretzschmar, K. et al. Profiling proliferative cells and their progeny in damaged murine hearts. Proc Natl Acad Sci U S A, published ahead of print December 7, 2018, doi.org/10.1073/pnas.1805829115 (2018).

66 Thum, T., Bauersachs, J., Poole-Wilson, P. A., Volk, H. D. \& Anker, S. D. The dying stem cell hypothesis: immune modulation as a novel mechanism for progenitor cell therapy in cardiac muscle. $J$ Am Coll Cardiol 46, 1799-1802, doi:10.1016/j.jacc.2005.07.053 (2005).

67 Tolar, J. et. al. The host immune response is essential for the beneficial effect of adult stem cells after myocardial ischemia. Exp Hematol. 35(4):682-90. (2007).

68 Francis Stuart, S. D., De Jesus, N. M., Lindsey, M. L. \& Ripplinger, C. M. The crossroads of inflammation, fibrosis, and arrhythmia following myocardial infarction. J Mol Cell Cardiol 91, 114-122, doi:10.1016/j.yjmcc.2015.12.024 (2016).

69 Chen, B. \& Frangogiannis, N. G. Immune cells in repair of the infarcted myocardium. Microcirculation 24, doi:10.1111/micc.12305 (2017).

70 Wynn, T. A. \& Vannella, K. M. Macrophages in Tissue Repair, Regeneration, and Fibrosis. Immunity 44, 450-462, doi:10.1016/j.immuni.2016.02.015 (2016).

71 Vieira, J. M. et al. The cardiac lymphatic system stimulates resolution of inflammation following myocardial infarction. J Clin Invest 128, 3402-3412, doi:10.1172/JCI97192 (2018). 
Figure Legends:

Figure 1 | Cardiac cell injection causes local inflammation with accumulation of distinct macrophage subtypes. a, Experimental scheme used here in $8 \mathrm{w}$-old male and female C57B//6J mice subjected to intra-cardiac injection of strain-matched bone marrow mononuclear cells (MNC), Alexa Fluor 594-conjugated zymosan (Zym.) or sterile saline (Sal.). Cells were isolated from Rosa26mTomato mice on the C57BI/ 6 background. b, Representative confocal immunohistochemistry micrographs of hearts from $n=4$ mice (Zym; $2 \mathrm{w}$-post) or $n=3$ mice (all other time points) showing activated CD68 macrophages (green) or the injected MNCs or zymosan (red). DAPI (blue) shows nuclei and dashed lines show injection sites. Data are from a minimum of 30 histological sections per mouse heart assessed from $n=4$ mice (Zym; $2 \mathrm{w}$-post) or $n=3$ mice (all other time points). Scale bars = $100 \mu \mathrm{m}$. c, Quantitation of $\mathrm{CD}^{+} 8^{+}$cells as a percentage of total cells $\left(\mathrm{DAPI}^{+}\right)$imaged at areas of injection from the groups shown in (b). ${ }^{*} p<0.05$ versus saline by one-way ANOVA with Tukey's posthoc test. Data are summarized as box and whisker plots indicating the median value (black bar inside box), 25th and 75th percentiles (bottom and top of box, respectively), and minimum and maximum values (bottom and top whisker, respectively). d, Experimental scheme using $8 \mathrm{w}$-old male and female Ccr2-RFP x Cx3cr1-GFP knock-in mice to simultaneously visualize CCR2 ${ }^{+}$and CX3CR1 ${ }^{+}$ macrophage subsets in vivo after injection of MNCs or zymosan. Here MNCs were isolated from wild-type C57B//6J mice while zymosan was not conjugated to a fluorophore. e, Representative confocal micrographs from MNC or zymosan-injected hearts, versus naïve (uninjected) controls (minimum of 30 sections assessed per mouse heart from $n=2$ naïve control mice and $n=3$ MNC or $n=3$ zymosan-injected mice), showing endogenous RFP and GFP immunofluorescence from CCR2 ${ }^{+}$ or $\mathrm{CX} 3 \mathrm{CR}^{+}$macrophages, respectively, at the injection site over a 7-day time course. Scale bars $=$ $100 \mu \mathrm{m}$. f, Distribution of CCR2 ${ }^{+}$and $\mathrm{CX} 3 \mathrm{CR} 1^{+}$macrophage subtypes in hearts at 3 days postinjection. Pie charts reflect the proportion of RFP $\left(\mathrm{CCR} 2^{+}\right)$or GFP $\left(\mathrm{CX} 3 \mathrm{CR} 1^{+}\right)$expressing macrophages, as well as $\mathrm{CCR} 2^{+} \mathrm{CX} 3 \mathrm{CR} 1^{+}$double-positive (yellow) macrophages detected by flow cytometry, as a percent of total macrophages identified by staining for F4/80 and CD64. Data are from $n=6 \mathrm{MNC}$ and $n=6$ zymosan-injected mice and $n=2$ naïve (uninjected) mice.

Figure 2 | Cell or inflammatory therapy induces some endothelial cell but not cardiomyocyte formation. a, Schematic outline of experiments performed in panels $\mathbf{b}$ and $\mathbf{c}$ in $8 \mathrm{w}$-old male and female C57BI/6J mice with intra-cardiac injection of MNCs, zymosan, or saline and analyzed $2 \mathrm{w}$ later. b, Representative cardiac immunohistochemistry for Ki67 (green) and PCM1 (purple) from MNCinjected hearts. DAPI (blue) shows nuclei. Scale bar $=100 \mu \mathrm{M}$. A minimum of 45 histological sections were analyzed per mouse heart from $n=4$ MNC-treated mice or $n=5$ for all other groups of mice. Yellow box denotes area shown in higher magnification insets on the right. Yellow arrowhead denotes a cardiomyocyte with cell cycle activity. Scale bars for inset images $=10 \mu \mathrm{m}$. c, Quantitation of cardiomyocytes with cell cycle activity $\left(\mathrm{PCM1}^{+} \mathrm{Ki}^{+} 7^{+}\right)$as a percentage of all cardiomyocytes imaged $\left(\mathrm{PCM1}^{+}\right)$at $6 \mathrm{~W}$. Data are from a minimum of 45 histological sections analyzed per mouse heart from $n=4 \mathrm{MNC}$-treated mice or $n=5$ for all other groups of mice. d, Schematic outline of experiments performed in panels (e-j) using c-Kit lineage tracing mice $\left(\mathrm{Kit}^{\mathrm{MerCreMer/+}} \times \mathrm{R}\right.$-eGFP) injected with MNCs, CPCs, zymosan or saline, then analyzed $2 \mathrm{w}$ or $6 \mathrm{w}$ later. Tamoxifen was administered continuously (in chow) starting one day before cell injection. e, Representative cardiac immunohistochemistry at $6 \mathrm{w}$ for $\alpha$-actinin (white) to show cardiomyocytes or eGFP (green) to show Kit allele-derived cells. DAPI (blue) shows nuclei. Scale bar $=50 \mu \mathrm{m}$. Yellow box highlights an eGFP ${ }^{+}$cardiomyocyte, shown at greater detail to the right. Inset scale bar $=10 \mu \mathrm{m}$. f, Quantitation of percent Kit allele-derived eGFP ${ }^{+}$cardiomyocytes relative to total cardiomyocytes counted. Data are from $n=3$ saline-treated mice or $n=5$ for all other groups of mice. $\mathbf{g}, \mathbf{h}$, Representative confocal cardiac immunohistochemistry images for $\mathrm{CD} 31^{+}$endothelial cells (white) and also showing MNCs (g) or zymosan (h) from injected hearts (red). Arrowheads denote CD31 ${ }^{+}$ 
endothelial cells that are also eGFP ${ }^{+}$. Scale bars $=100 \mu \mathrm{m}$. i, j, Quantitation of percent eGFP ${ }^{+}$ endothelial cells relative to total endothelial cells counted, either $2 w$ (i) or $6 w$ (j) post-injection. Data are from $n=6 \mathrm{Sal} / 2 \mathrm{w}$ or Zym/6w or $n=5$ all other groups of mice. * $p<0.05$ by one-way ANOVA with Tukey's post-hoc test. All numerical data are summarized as box and whisker plots. Data and representative micrographs in e-j are from a minimum of 45 histological sections analyzed per individual mouse heart from the numbers of mice as indicated above.

Figure 3 | Cell or inflammatory therapy rejuvenates post-I/R heart function. $a$, Schematic outline of experiments performed in panels $\mathbf{b}$-c in which $8 \mathrm{w}$-old male and female C57B//6J mice received 120 min of myocardial ischemia followed by reperfusion (I/R) injury then intra-cardiac injection 1w later of MNCs, CPCs, zymosan, or sterile saline flanking the injury area, followed by analysis $2 \mathrm{w}$ or 8 w later. b-c, Fractional shortening (FS) as measured by echocardiography in the groups indicated, 2w post-cell or zymosan therapy (b) or 8w post-cell or zymosan therapy (c). ${ }^{*} p<0.05$ vs Sham/Sal, or ${ }^{\#} p<0.05$ vs I/R/Sal. by one-way ANOVA with Dunnett's post-hoc test. The same sham group is shown in $\mathbf{b}$ and $\mathbf{c}$ as these experiments were performed in parallel. $\mathbf{d}, \mathrm{FS}$ as measured by echocardiography in male and female post-I/R mice that received cyclosporine A (CsA; $15 \mathrm{mg} / \mathrm{kg}$ body weight/d) delivered by osmotic minipump, starting one day before MNC or saline injection and continuing out for $2 w$ post-injection. ${ }^{*} p<0.05$ vs Sham/Saline by one-way ANOVA with Dunnett's post-hoc test. e, Confocal micrographs at the infarct border zone of hearts from male and female Ccr2-RFP x Cx3cr1-GFP knock-in mice ( $n=2$ mice per group and timepoint with a minimum of 10 sections assessed per mouse heart) at either 3 days or 2 weeks post-I/R. g, Schematic outline of experiments performed in panels (h) and (i) in male and female $\mathrm{Ccr}^{-/-}$or $\mathrm{Cx} 3 \mathrm{cr}^{-/-}$mice in the C57BI/6 background that were subjected to I/R then injected with MNCs or sterile saline $1 \mathrm{w}$ later. $\mathbf{h}$, $\mathrm{FS}$ in $\mathrm{CCr}^{-/-}$or $\mathrm{C} \times 3 \mathrm{cr}^{-/-}$mice or wild-type C57B//6 mice $3 \mathrm{w}$ post-I/R (2w post-cell injection). ${ }^{*} \mathrm{p}<0.05$ vs Pre-l/R (Pre) or ${ }^{\#} p<0.05$ vs I/R/Sal. by one-way ANOVA with Tukey's post-hoc test. i, Quantitation of $\mathrm{CD}^{+} 8^{+}$cells as a percentage of total cells (DAPI ${ }^{+}$) imaged at the infarct border zone, 3w post-I/R. ${ }^{*} p<0.05$ versus WT/Saline or ${ }^{\&} p<0.05$ versus $C c r 2^{-/ /} /$Sal. by one-way ANOVA with Tukey's post-hoc test. The number $(n)$ of mice in all experimental groups is indicated below or within the respective plot. All numerical data are summarized as box and whisker plots.

Figure 4 | Cell therapy benefits the mechanical properties of the infarct via remodeling of the extracellular matrix. a, Schematic outline of experiments performed in panels $\mathbf{b}$-e in this figure. $\mathbf{b}$, Representative picrosirius red-stained cardiac histological images from the infarct border zone of 3w post-I/R mice subjected to MNC or saline injection. Fibrosis is shown in red. Scale bars $=100 \mu \mathrm{m}$. Images and quantitation in (c) are from $n=5$ saline-treated, $n=12$ MNC-treated, or $n=7$ freeze-killed MNC-treated mice, with a minimum of 20 histological sections assessed from each individual mouse heart. c, Quantitation of fibrotic area at the infarct border zone in MNC, freeze thaw-killed MNC, or saline-treated hearts (number of mice in each group shown below each box and whisker plot), 3w post-I/R. ${ }^{*} p<0.05$ versus I/R/Saline by one-way ANOVA with Tukey's post-hoc test. d, Change in passive force generation over increasing stretch-lengthening (percent of $L_{0}$ ) in isolated infarct strips from MNC or saline-treated hearts, 3w post-I/R. ${ }^{\star} p<0.05$ versus I/R/Saline by Student's 2-tailed t-test. e, Gene expression levels by RT-PCR for selected extracellular matrix (ECM) and matrix-associated genes in isolated infarct strips from MNC or saline-treated hearts, $3 \mathrm{w}$ post-I/R. * $\mathrm{p}<0.05$ versus I/R/Saline by Student's 2-tailed t-test. f, Representative confocal micrographs of pre-fabricated collagen patches that were seeded and cultured for 5 days with either bone marrow-derived macrophages (BMDM) or peritoneal macrophages (PM) isolated from wild-type male and female mice, versus cell-free control patches cultured in media. Fluorescence signal is from second harmonic generation microscopy using $840 \mathrm{~nm}$ light to allow for specific detection of native type I and II collagen. Scale bars $=100 \mu \mathrm{m}$. g, Schematic outline of experiments using activated cardiac macrophages isolated from post-I/R Ccr2-RFP x Cx3cr1-GFP knock-in mice using fluorescence 
activated cell sorting (FACS). $\mathrm{CCR} 2^{+}$and $\mathrm{CX} 3 \mathrm{CR} 1^{+}$macrophages were then cultured with isolated cardiac fibroblasts for 72 hrs. h-k, Fibroblast mRNA was then used for RT-PCR to assess expression of smooth muscle a-actin (Acta2 / aSMA, h), lysyl oxidase (Lox, i), connective tissue growth factor $($ Ctgf, j) or collagen 1 alpha 2 (Col1a2, k). Numerical data in (d) are presented as the mean + SEM from the number $(n)$ of mice indicated in the figures. All other numerical data are summarized as box and whisker plots. Micrographs in $\mathbf{f}$ are representative of five different collagen patches seeded with cells pooled from $n=4$ mice ( 2 male and 2 female). Data in $\mathbf{g - k}$ are from five replicates generated over fibroblasts isolated from $n=10$ wild-type mice ( 6 male and 4 female) and macrophages isolated from $n=6$ Ccr2-RFP $\times$ Cx3cr1-GFP knock-in mice (3 male and 3 female). 
a Zymosan-Alexa594

.. Injection 3d-post 2w-post

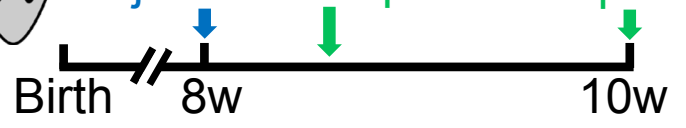

h Saline

C

Fig 1

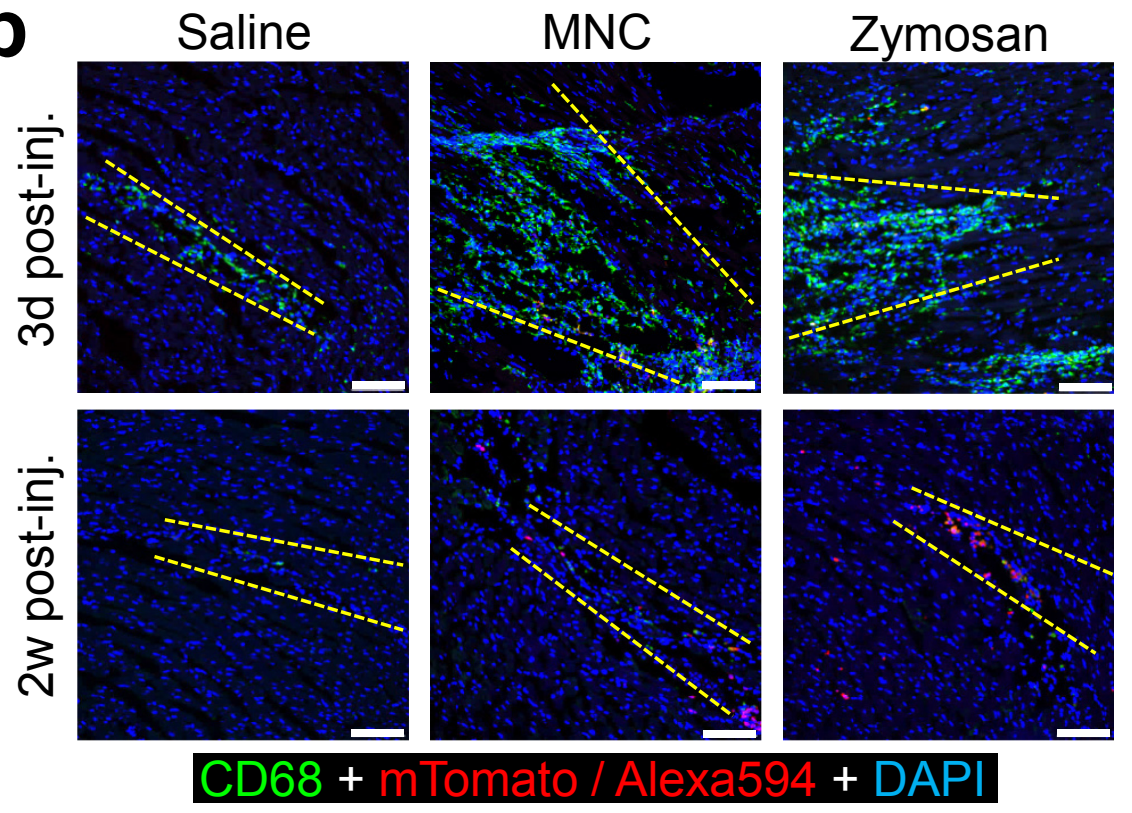

d

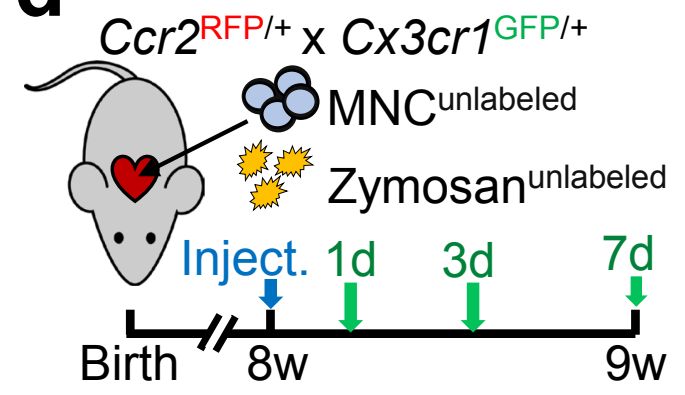

f $\underline{\mathrm{Ccr} 2^{\mathrm{RFP} /} \times \mathrm{C} \times 3 \mathrm{Cr} 16 \mathrm{FF} / \mathrm{t}}$ $\square \mathrm{GFP}^{+} \square \mathrm{RFP}^{+} \square \mathrm{RFP}^{+}$ Naive MNC Zym. 4 10

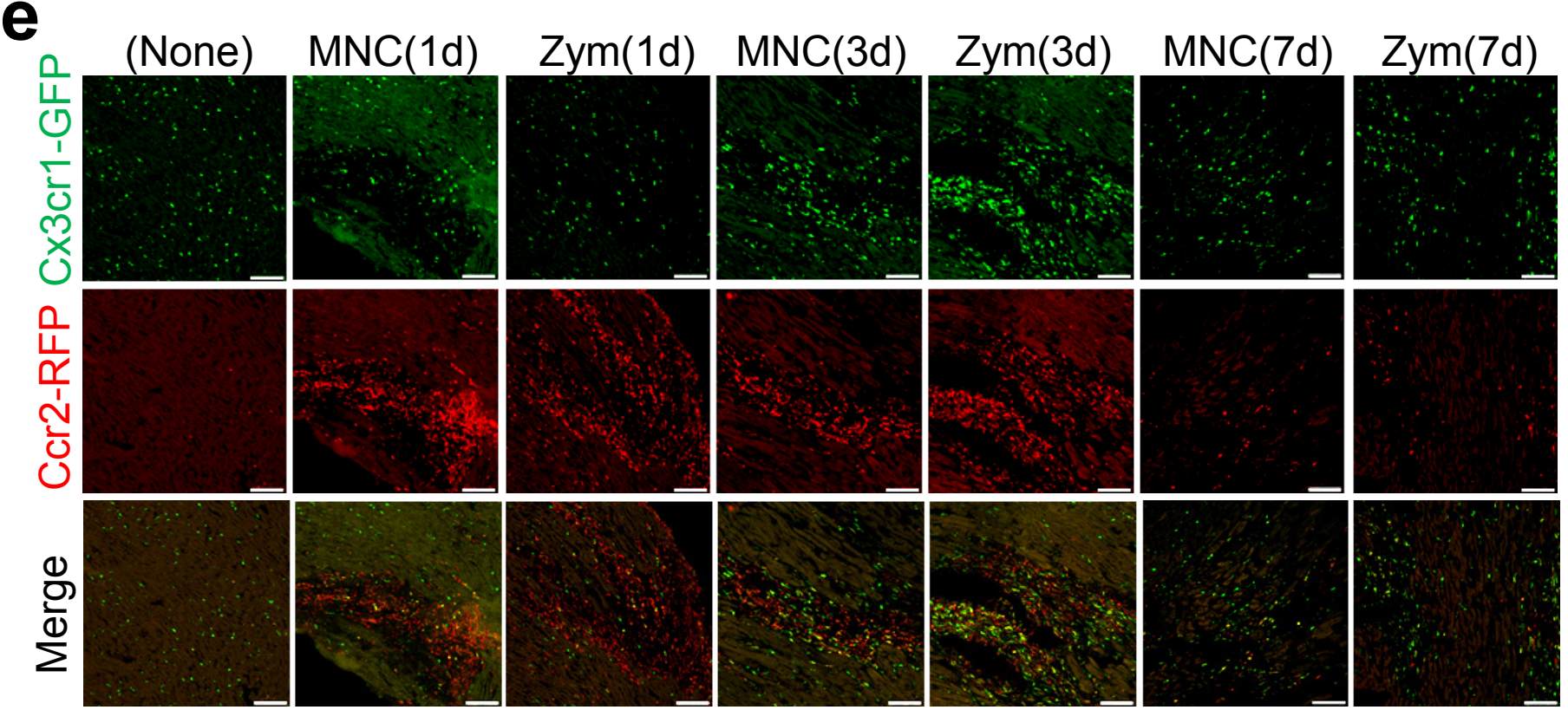


?.. Injection

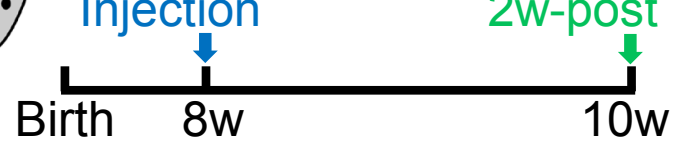

d $\mathrm{Kit}^{+/ \mathrm{MCM}} \times \mathrm{R} 26-\mathrm{eGFP}$

\&MNC-R26-mTomato of CPC-R26-mTomato Zymosan-Alexa594 Injection 2w-post 6w-post

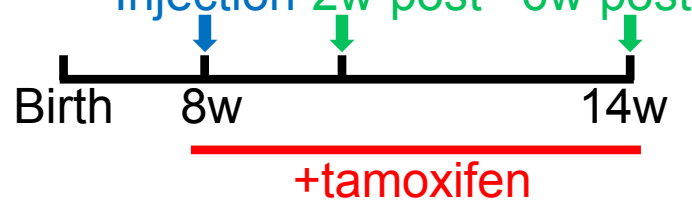

+tamoxifen b

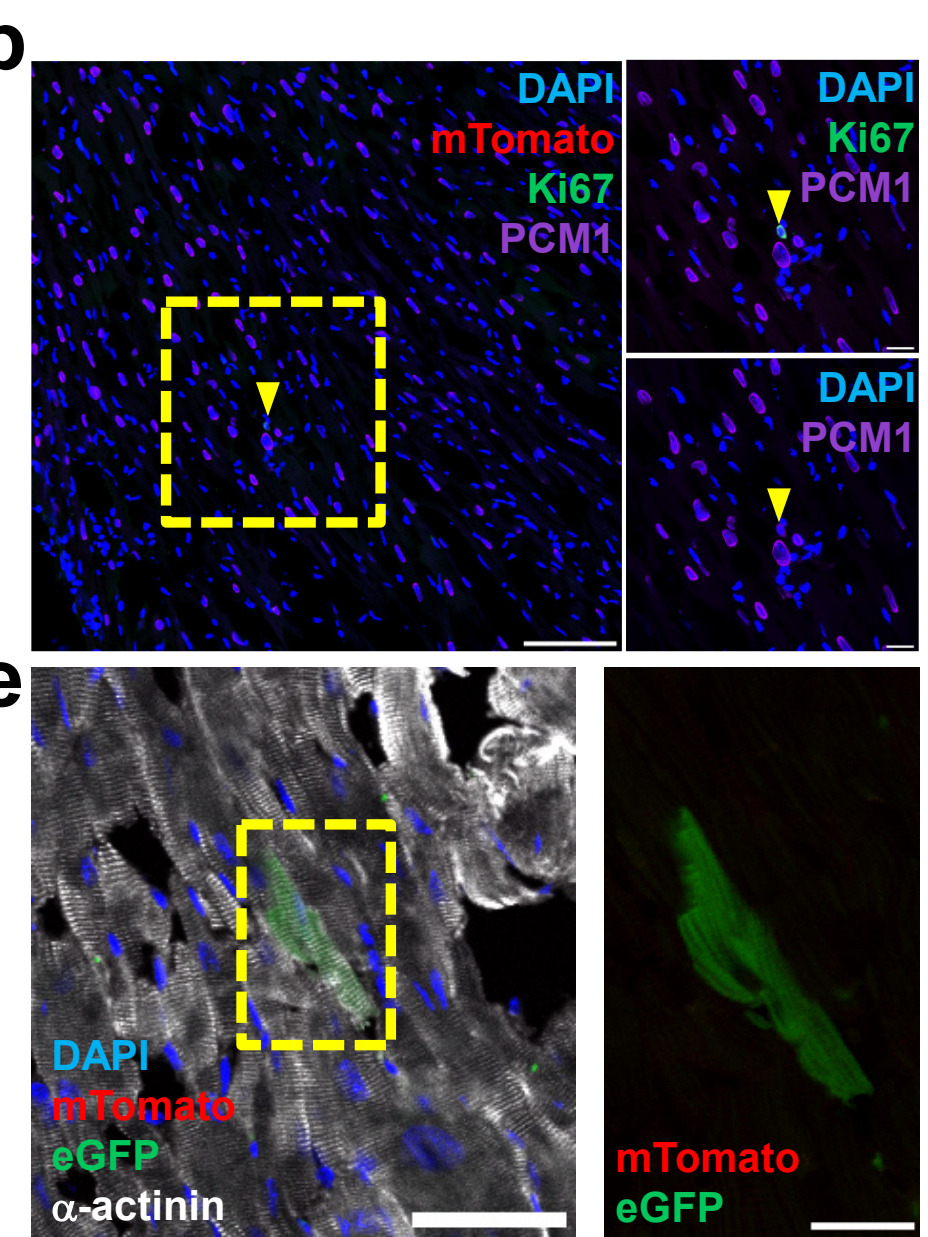

C

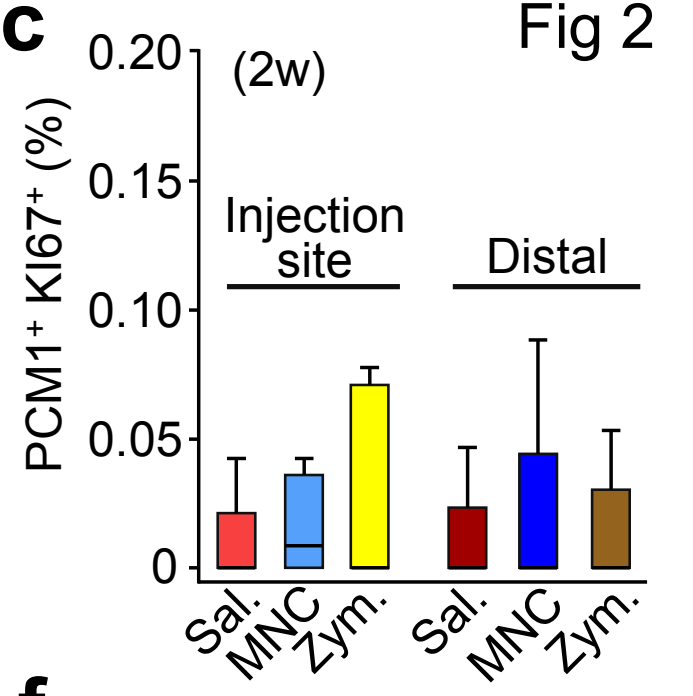

f

ஓ 0.20$](6 w)$

\& 0.15

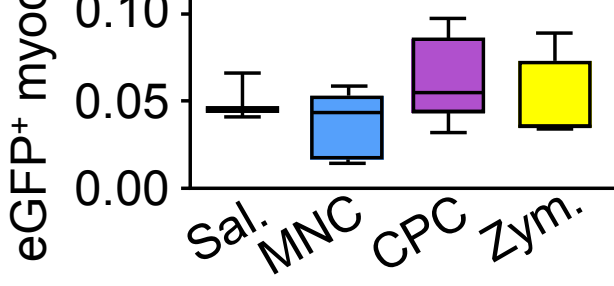

9)

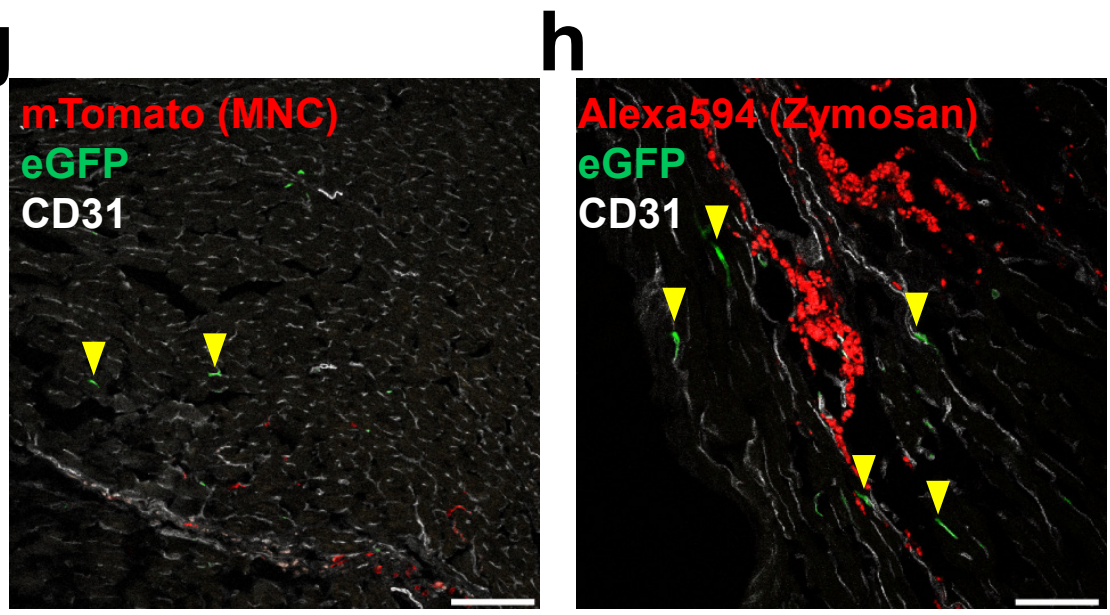

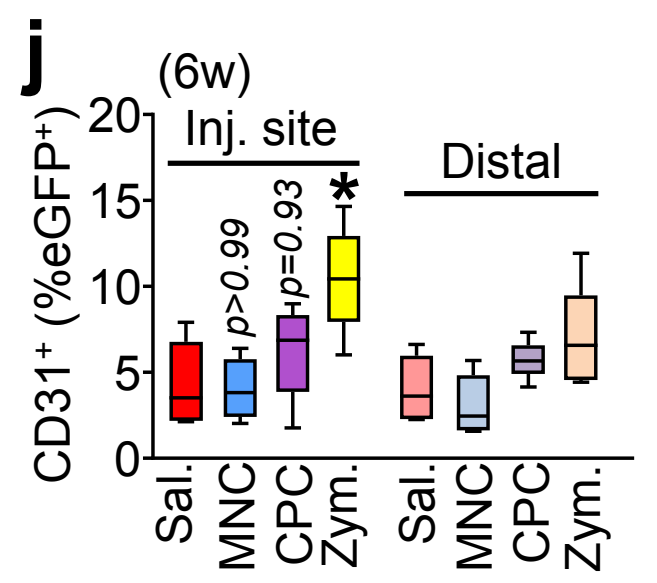


